\title{
La des-materialización de productos tangibles en una perspectiva de sustentabilidad
}

\section{Nuri Barón, Gabriela}

Resumen: En el ejercicio de pronóstico de escenarios futuros para un desarrollo sostenible, los paradigmas occidentales de libre mercado se están viendo profundamente amenazados por nuestros límites ecológicos. Ahora está claro que la crisis ambiental está directamente relacionada con el consumo y no puede abordarse con respuestas técnicas tradicionales. Las innovaciones más prometedoras que presentan soluciones viables a la crisis ambiental son de naturaleza social. Dentro de esta perspectiva, los diseñadores parecen haber llegado a un callejón sin salida en relación a la creación material. Lejos de desalentar, estos límites han arrojado luz sobre todo un nuevo espectro de

Cuadernos del Centro de Estudios de Diseño y Comunicación №70

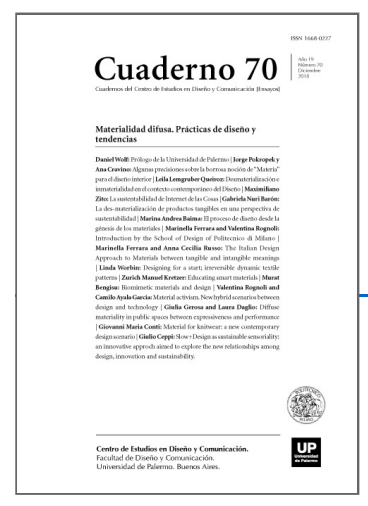

ISSN: 1668-0227

Materialidad difusa.

Prácticas de diseño y

tendencias

Año XIX, Diciembre 2018, Buenos Aires, Argentina | 172 páginas

descargar PDF

ver índice de la publicación

Ver todos los libros de la publicación

compartir en Facebook

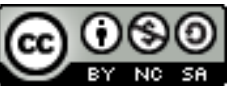

Esta obra está bajo una Licencia Creative Commons Atribución-NoComercialCompartirlgual 4.0 Internacional oportunidades profesionales hacia el bienestar social, donde los diseñadores tienen el liderazgo como facilitadores creativos. La desmaterialización se convierte en un curso de acción desafiante, atractivo y viable, colocando a las personas como el material principal para el cambio.

Palabras clave: servicización, colaboración, inacabado, enfoque sistémico, bienestar, consumo, desmaterialización, desarrollo sostenible.

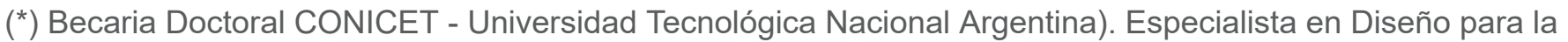
Sustentabilidad e Innovación Social. Diseñadora Industrial graduada en Argentina, realizó un master en el Politécnico de Milán realizando proyectos en Argentina, Italia y Asia.

Introducción

Cuando los diseñadores proyectan escenarios futuros en relación a materiales nuevos, una deslumbrante serie de innovaciones despierta nuestro espíritu creativo y estimula nuestra mente con oportunidades ilimitadas. Pero 
solo una mirada al estilo de vida actual es suficiente para comprender lo difícil que es conectar inocentemente la palabra futuro con la palabra material. Las oportunidades solo parecen ser ilimitadas. Resulta ingenuo y peligroso para los diseñadores visualizar futuros sin tener en cuenta nuestros propios límites naturales. No será necesario citar la vasta cantidad de datos científicos que describen el estado de emergencia que este mundo está atravesando. Como sociedad somos partícipes de un deterioro ambiental que nos llevará a la extinción como raza. De acuerdo a las Naciones Unidad, para el año 2030 necesitaremos "dos planetas" de recursos para sostener la población mundial (Moore et al., 2012). Esta crisis ambiental está estrictamente ligada a una crisis social y ética de inequidad, ya que unos pocos países consumen la mayoría de los recursos ( $20 \%$ de la población consume $80 \%$ de los recursos mundiales), mientras las consecuencias ecológicas se sufren en todo el planeta (FAO, 2003).

Se justifica decir que la culpa la tienen los patrones de consumo predominantes. Aquí es donde el rol tradicional del Diseñador Industrial es profundamente desafiado, ya que la base de su labor es la concepción de productos para ser industrialmente producidos. Numerosas aproximaciones por parte del diseño han surgido como respuesta al problema de la sustentabilidad, pero hay preguntas fundamentales que debemos hacernos: Cuál es el rol ético del diseñador en una sociedad híper-consumista? Dónde recae el peso ético de decidir cuales productos contribuyen a solucionar la crisis y cuales contribuyen al problema? El diseño, al estar profundamente ligado a la producción y al consumo, juega un papel crucial en la transición hacia una sociedad más sustentable. (Martilla, 2011)

\section{Des-materialización y consumo}

La idea de desmaterialización implica una reducción material, de energía, agua y tierra para obtener iguales (o mejores) resultados económicos. De acuerdo al Programa Ambiental de Naciones Unidas (UNEP, 2011):

La Desmaterialización describe principalmente la reducción de requisitos materiales de economías en su totalidad. Requiere de (a) la reducción de intensidad material de productos y servicios (ej. aumentar la eficiencia material) y (b) la reducción de recursos materiales primarios (ej. El cambio a una economía circular).

A través del diseño y la innovación en productos y procesos, basado en el "Pensamiento de Ciclo de Vida" (EU, 2010), los productores, proveedores y diseñadores están aprendiendo a hacer más con menos: usar menos material, extender la vida de los materiales, usar procesos con menor intensidad energética para transformar estos materiales, y finalmente cuestionar si ese producto material brinda la mejor solución a la necesidad que lo legitima. En este último punto, en el nivel más profundo, el concepto de des-materialización lleva a menos consumo y la consecuente menor demanda de productos. Así se llega a la pregunta clave: Cuando la mayoría de los productos comercializables no sólo son innecesarios, sino materialmente inviables en un futuro realista, cuál es el rol del Diseñador Industrial? La respuesta a esta pregunta requiere que de una dura y crítica mirada a la concepción tradicional de nuestra profesión, sin pasar por alto las consecuencias éticas que están en juego. Numerosos autores han reflexionado acerca de esta problemática en particular y han brindado análisis crítico, marco metodológico y herramientas que amplifican el rol del diseñador, agregando valor a sus capacidades técnicas, creativas y comunicacionales. A lo largo de este artículo se presentan algunos de estos enfoques, colocando la des-materialización como centro estratégico para un desarrollo sustentable. 
A través de un enfoque sistémico, la des-materialización se puede obtener mediante técnicas que abordan desde el producto en sí hasta las dimensiones culturales y sociales en sus niveles más profundos. El pensamiento sistemático fue introducido por el profesor Jay Forrester en 1956 para la comprensión de sistemas sociales complejos (Forrester, 1994). En vez de separar el objeto de estudio en partes más pequeñas (como lo hace el análisis tradicional), el pensamiento de sistemas observa las interacciones de las diferentes piezas del objeto estudiado, expandiendo el alcance con el propósito de integrar todas sus relaciones. Dentro de este marco, el diseño adquiere un mayor potencial para resolver temas ambientales desde la etapa proyectual. Un ejemplo puede ser la exitosa adaptación de herramientas de Análisis de Ciclo de Vida (ACV) en el "Diseño para la Sostenibilidad". (Vezzoli y Manzini, 2008)

Tukker (2004) propone niveles de intervención que van desde los aspectos materiales, como "mejora del impacto de la eficiencia tecnológica" (energías limpias) y producción eficiente (menor consumo energético en la producción), tratan también los aspectos sociales como la planificación de la intensidad de uso (uso compartido, uso público) y el aumento de funcionalidades inmateriales, hasta la creación de contextos sin necesidades. Se llega a la conclusión de que la tarea de adoptar una sustentabilidad verdadera ya no es predominantemente científica o tecnológica, sino cultural y social. (Hamilton, 2010)

Similarmente, Vezzoli y Manzini (2008), en su libro Design for Environmental Sustainability, presentan cuatro niveles de intervención donde la des-materialización se logra de la siguiente manera:

1. El re-diseño ambiental de sistemas existentes: este nivel implica la reducción de materia y energía incluidas en el producto. Existen varias metodologías publicadas que actúan en este nivel, que generalmente se aplican a diferentes etapas de su ciclo de vida. Como un ejemplo, se podría pensar en una botella de agua descartable/reciclable realizada con menor cantidad de plástico debido al re-diseño de su forma estructural mediante paredes más finas de material. Este re-diseño no sólo refleja el menor uso de materia prima, también el peso del producto se reduce, necesitando menor energía para su transporte, así como su forma puede facilitar la compactación, significando menos volumen de almacenamiento como residuo, etc. Este nivel no requiere un cambio en los patrones de consumo y la des-materialización sólo ocurre a niveles técnicos.

2. El diseño de nuevos productos y servicios: Este nivel implica el remplazo de los productos existentes con nuevos productos concebidos para ser ambientalmente eficientes en cada etapa de su ciclo de vida. Aunque también se trabaja en un nivel técnico, las propuestas resultantes tienden a experimentar dificultades para encajar en los patrones de consumo existentes y requieren de un cambio en los valores y el contexto cultural del usuario. Siguiendo con el ejemplo de la botella de agua, este nivel se ilustra con las botellas de plástico rígido concebidas para su rellenado y re-uso. La botella en si misma posee más intensidad material para su durabilidad, pero la des-materialización es alta considerando la extensión en su vida útil.

3. El diseño de nuevos Sistemas de Productos y Servicios: En este nivel se cambia el foco desde el diseño del producto en sí mismo hacia la satisfacción de la necesidad que motiva la existencia del producto. Este enfoque le permite al diseñador realizar propuestas radicalmente diferentes (e intrínsecamente sustentables) que satisfacen la misma necesidad. La des-materialización sucede, no sólo a nivel de ciclo de vida de todos los productos y servicios involucrados, sino también en cambios de comportamiento de los actores participantes. Para que estas propuestas sean efectivas deben ser social y culturalmente apropiadas (principalmente a través 
de la comunicación estratégica). Este nivel implica una des-materialización radical a través de cambios profundos en patrones de consumo, tales como el uso compartido.

4. La propuesta de nuevos escenarios de estilos de vida sustentables: este nivel trabaja directamente en la reorientación de los patrones de producción y consumo dominantes. La des-materialización se logra a través de elecciones de vida subsecuentes, que se promueven mediante el escenario propuesto. Los escenarios operan en la esfera cultural ya que se basan en la investigación, colección y re-interpretación de casos (exitosos y prometedores) existentes. Este nivel reconoce la transversalidad de la problemática y sus profundas raíces sociales, trabajando no sólo la faceta ambiental del Diseño Sustentable, sino también sus implicaciones sociales y económicas. Como ejemplo de este nivel, el consumidor cuestionaría críticamente la forma en la que se comercializa el agua embotellada (y sus consecuencias ambientales), renunciaría a ser parte de ese sistema y participaría activamente (co-creación) en la propuesta de una alternativa de consumo de agua.

Los autores resumen brillantemente los niveles mencionados diciendo que "el Diseño para la Sustentabilidad Ambiental implica facilitar la respuesta a la demanda social de bienestar por parte del sistema de producción, reduciendo drásticamente la cantidad de recursos ambientales necesarios para el sistema actual" (Vezzoli y Manzini, 2008). En síntesis, ellos proponen la des-materialización del bienestar.

Es importante resaltar que las estrategias mencionadas no implican una pérdida de bienestar por parte del usuario, sino un cambio en los valores que definen el bienestar en sí mismo. En este contexto, la crisis ambiental y social ha llegado a tales profundidades que la gente está comenzando a cuestionarse la creencia base que establece que la mejora del bienestar depende del crecimiento ilimitado del nivel de consumo (Hamilton, 2010). Como ejemplo de este cambio ecológico profundo en el comportamiento, aparecen fenómenos sociales como el downshifting, movimiento en el que los adeptos toman la decisión voluntaria de reducir los ingresos y el consumo propios, a favor de una mayor calidad de vida. Estos innovadores sociales comprenden que poseer más tiempo para actividades que mejoran su nivel de bienestar (que generalmente involucran a la familia, amigos, salud, pasatiempos, etc.) es más satisfactorio que destinar el tiempo a trabajar para ganar dinero para comprar cosas que prometen aumentar su bienestar.

Des-materialización mediante in-terminación y colaboración

Una profunda reflexión de la evolución del diseño puede realizarse al analizar la evolución de los sistemas naturales. Una aproximación complementaria, que observa a la naturaleza con el propósito de encontrar estrategias evolutivas de adaptación a la crisis ecológica, es la de admisibilidad del error o "Error-Friendliness" (Von Weizaker, 1988). Este concepto está íntimamente ligado al diseño para la sustentabilidad, en el sentido de la concepción de productos con ciclos de vida largos. Ezio Manzini (1994) elabora sobre el concepto de admisibilidad del error, al criticar los productos super-especializados que no admiten versatilidad o evolución temporal. Mientras la super-especialización frecuentemente lleva a la extinción en contextos de cambio radical, los productos sub-óptimos habilitan su adaptación por parte de los usuarios y sobreviven a cambios contextuales, extendiéndose su vida útil.

Una estrategia para la admisibilidad del error es proyectar productos que no estén "terminados", lo que llamaremos in-terminación. Los productos no-terminados se transforman en una herramienta que permite al usuario co-crear el producto finalizado que satisfaga su necesidad. La clave se encuentra en la versatilidad. Un 
claro ejemplo podría ser un juego de ladrillos Lego: desde su invención han jugado con ellos generaciones y generaciones de niños de un amplio rango de edades, independientemente de su género, y al día de hoy se utilizan por adultos como herramientas de Design Thinking, etc. Es un juguete durable y versátil que sobrevive a las modas. En un ejemplo más complejo se podría pensar en un sistema modular de equipamiento para oficinas que puede mutar, adaptarse y cambiar en el tiempo. Los beneficios ambientales son numerosos: extensión de la vida útil del sistema, reposición por partes para su reparación, posibilidades de expansión, consumo compartido, personalización y demás. En síntesis, los sistemas tecnológicos basados en soluciones modulares, descentralizados y diversos en su producción y funcionamiento, intrínsecamente admiten mayor grado de error. (Manzini, 1994)

A nivel de satisfacción del consumidor, los productos no-terminados están abiertos a la colaboración, participación y co-creación, con todos los beneficios de la auto-organización. Cuando un sistema de productos es versátil, permite diferentes combinaciones, completándose con las capacidades de los usuarios. Los resultados pueden ser inesperados, pueden evolucionar o mutar en el tiempo, pueden ser resilientes a los errores y socialmente innovadores. Un buen ejemplo de esto es el movimiento de software de código abierto, que ha llevado a resultados sin precedentes como Wikipedia o Linux.

\section{Des-materialización mediante servificación}

La des-materialización puede suceder a una escala mayor si uno se mueve desde una economía basada en los productos a una economía basada en los servicios. La economía de los servicios se basa en la optimización del uso de bienes y servicios, y por lo tanto en la gestión de la riqueza existente (bienes, conocimiento, recursos). El objetivo económico de la economía de servicios es "crear el valor de uso más alto durante el mayor tiempo posible, consumiendo la menor cantidad de recursos energéticos y materiales posibles" (Stahel, 2001). Si un consumidor no poseyera un producto y lo alquilara, un productor posee el incentivo de diseñar productos durables, reparables, actualizables y reusables. (Dooley, 2013)

Las empresas se están dando cuenta de que aunque vendan productos, la gente compra el servicio que éstos satisfacen. Se dan cuenta de que los usuarios compran productos para satisfacer necesidades que requieren de otros productos y servicios para brindar satisfacción, y que poseen necesidades emocionales que van más allá de lo que el producto puede brindar. Al completar su oferta con servicios que agregan valor al producto, refuerzan, habilitan, educan y expanden la experiencia del producto y crean una economía del sentido. Estas compañías pueden ser con o sin fines de lucro, parte de un modelo económico social o no. Su valor agregado se financia en el precio y a través de las transacciones repetidas con los consumidores, que se fidelizan mediante el servicio (Oliva and Kallemberg, 2003). La base de este comercio se vuelve la relación y no la transacción. Las estrategias de servificación aumentan la eficiencia material del modelo de producción y consumo, habilitando beneficios sociales que van más allá de la des-materialización.

Finalmente y de mayor importancia, está el hecho de que los servicios se han tornado una fuente sustentable de ventaja competitiva al hacer uso del recurso más abundante del planeta: el trabajo humano.

\section{Conclusiones}


Las tecnologías y materiales del futuro posibilitan nuevas perspectivas para la des-materialización a nivel de producto, donde la liviandad de un nuevo producto puede reducir su impacto ambiental. Sin embargo, en un mundo finito materialmente, que enfrenta una crisis ambiental sin precedentes, los diseñadores necesitan ampliar su rol profesional para satisfacer las necesidades sociales con propuestas de radicalmente menor intensidad material. El diseño industrial como profesión se enfrenta actualmente a una gran crisis ética, ya que su propósito es la satisfacción de necesidades humanas (en ambos niveles, el técnico y el emocional) mediante un proceso continuo de diseño y re-diseño, que implica producción, comercialización y compra repetida. Mientras que la base de esta crisis ambiental es el modelo de producción-consumo en el que la profesión del diseñador se basa, un escenario futuro optimista implica la elección de consumir menos en pos de una mayor calidad de vida, desafiando este modelo.

No es una conclusión personal cuando afirmo que la única perspectiva viable es la de cambio radical e innovación sistémica, numerosos autores han llegado a la misma drástica y desafiante conclusión. En este escenario, los diseñadores poseen las herramientas y el conocimiento para actuar como promotores de nuevas interpretaciones del bienestar basadas en menor consumo de objetos materiales y mayor cantidad de valor inmaterial. Consecuentemente, se han propuesto numerosas estrategias de diseño que proponen la desmaterialización en diferentes niveles, desde el nivel de producto al nivel de comportamiento. La servificación y la interminación se vuelven estrategias prometedoras para la reducción drástica de intensidad material en los productos. Estas aproximaciones poseen un fuerte contenido social, ya que enfatizan la interacción ampliada (y sistémica) entre los actores, la participación de los usuarios y la distribución abierta de los resultados. Aquí, los roles del diseñador, del productor y del consumidor son más permeables, con límites borrosos que habilitan formas altamente innovadoras de hacer las cosas. Estas nuevas formas pueden necesitar nuevas herramientas, pero se basan mayormente en la comunicación estratégica y la habilidades creativas para la resolución de problemas.

No se puede concebir visiones del futuro material sin considerar los límites de nuestros recursos materiales. Los límites son parte de nuestro ecosistema y cualquier sistema concebido para su crecimiento ilimitado (como el sistema capitalista) es insostenible y lleva al colapso. Los límites materiales son oportunidades para liberarnos de paradigmas tradicionales, abrirnos a nuevos roles profesionales hacia nuevas posibilidades de intervención. La des-materialización estratégica se propone como un camino viable, desafiante y atractivo hacia un futuro sostenible. Los diseñadores debemos provocar a los paradigmas existentes, e innovar colocando a la gente como el material primario para el cambio. Como dijo Ezio Manzini: en un planeta superpoblado, el recurso humano es el más abundante.

\section{Referencias}

Dooley, K. (2013). Product design: Do it with Dematerialization. CSR Wire, 14-16. Retrieved from: http://www.csrwire.com/blog/posts/1078-product-design-do-it-withdematerialization

EU - European Union (2010). Making sustainable consumption and production a reality. ISBN: 978-92-79-143571 DOI: 10.2779/91521. Available at http://bookshop.europa. eu/en/making-sustainable-consumption-andproduction-a-reality-pbKH3109259/ 
FAO - Food and Agriculture Organization of the United Nations (2003). The state of food insecurity in the world. ISBN 92-5-104986-6. Rome, Italy.

Forrester, J. W. (1994). System dynamics, systems thinking, and soft OR. Syst. Dyn. Rev., 10: 245-256. doi:10.1002/sdr.4260100211

Hamilton, C. (2010). Consumerism, self-creation and prospects for a new ecological consciousness. Journal of Cleaner Production, 18(6), 571-575. http://doi.org/10.1016/j. jclepro.2009.09.013

Manzini, E. (1994). Physis and design. Interaction between nature and culture. Article available at: https://www.researchgate.net/publication/39085531. Retrieved on: 02 August 2016

Marttila, T. (2011). Dematerialization by Design - Sustainability in the consumer society. Position paper for ARKTIS Summer School 2011. Design Connections Doctoral Program. Aalto University, School of Art and Design.

Moore, D.; Galli, A.; Cranston, G. R. and Reed, A. (2012). Projecting future human demand on the Earth's regenerative capacity. Ecological Indicators, 16, 100-112. http://doi. org/10.1016/j.ecolind.2011.06.017

Oliva, R. and Kallenberg, R. (2003). Managing the transition from products to services. International Journal of Service Industry Management, Vol. 14, No. 2, pp. 160-172.

Stahel W. (2001). Sustainability and Services, in Sustainable Solutions - Developing products and services for the future, Charter, M. and Tischner, U. (edit by), Greenleaf publishing, UK.

Tukker, A. (2004). Eight types of product-service system: eight ways to sustainability? Experiences from suspronet, Business Strategy and the Environment, vol. 13, no. 4

UNEP (2011). Prepared by Meghan O'Brien and Stefan Bringezu, Wuppertal Institute. Draft Glossary of Terms Used by the International Resource Panel. Page 3.

Vezzoli, C. (2007). System Design for Sustainability. Milano: Maggioli Editore.

Vezzoli, C., \& Manzini, E. (2008). Design for Environmental Sustainability. London: Springer London. http://doi.org/10.1007/978-1-84800-163-3.

Weizaker, E. i C., Von (1988). Come vivere con gli errori. Il valore evolutivo degli errori. Ceruti, M., Laszlo, E.

Abstract: In the practice of future scenario forecasting for a sustainable development, the western free market paradigms are becoming deeply threatened by our ecological limits. It is clear now that the environmental crisis is directly linked to consumption and cannot be tackled with traditional technical responses. The most promising innovations that present viable solutions to the environmental crisis are of social nature. Within this perspective, Designers seem to have arrived to a dead-end road in relation to material creation. Far from discouraging, these limits have shed light on a whole new spectrum of professional opportunities towards social-well-being, where 
Designers have the lead as creative facilitators. Dematerialization becomes a challenging, attractive and viable course of action, placing people as the primary material for change.

Keywords: servicization - collaboration - unfinishedness - systemic approach - well-being - consumption dematerialization - sustainable development.

Resumo: No exercício de prever cenários futuros para o desenvolvimento sustentável, os paradigmas ocidentais do livre mercado estão sendo profundamente ameaçados pelos nossos limites ecológicos. É agora claro que a crise ambiental está diretamente relacionada com o consumo e não pode ser tratada com respostas técnicas tradicionais. As inovações mais promissoras que apresentam soluções viáveis para a crise ambiental são de natureza social. Nessa perspectiva, os designers parecem ter chegado a um impasse em relação à criação material. Longe de desanimar, esses limites lançaram luz sobre todo um novo espectro de oportunidades profissionais para o bem-estar social, onde os designers têm liderança como facilitadores criativos. A desmaterialização se torna um campo de ação desafiador, atraente e viável, colocando as pessoas como o principal material para a mudança.

Palavras chave: servicização - colaboração - inacabado - abordagem sistêmica - bem-estar - consumo desmaterialização - desenvolvimento sustentável.

La des-materialización de productos tangibles en una perspectiva de sustentabilidad fue publicado de la página 45 a página52 en Cuadernos del Centro de Estudios de Diseño y Comunicación №70 